\title{
Components of a Wind Tunnel Balance: Design and Calibration
}

\author{
Miguel A. González, José Miguel Ezquerro, Victoria Lapuerta, \\ Ana Laverón, and Jacobo Rodríguez \\ Escuela Técnica Superior de Ingenieros Aeronáuticos \\ Universidad Politécnica de Madrid \\ Spain
}

\section{Introduction}

The aim of wind tunnel tests is the simulation of the flow around bodies or their scaled models. In aeronautical applications, the measurement of aerodynamic loads in a wind tunnel, forces and momentums, is a very difficult task due to the required accuracy. The wind tunnel balances, comprised by several hardware and software components, provides directly the pursued measurements, with high accuracy and reliability. For these reasons, among others, wind tunnel balances have become a common tool in testing facilities.

This chapter starts with a general description of wind tunnel balances. The number of measuring components and the position of the balance with relation to the model and wind tunnel chamber determine the wind tunnel balances designs. The most flexible ones, in terms of usability, are the six components external balances, so these will be referenced for introducing the calibration process, which is one of the key points to achieve the required aerodynamic tests results accuracy and reliability. Because of its influence on the drag measurement accuracy, the coupling effect between lift and drag measurements is analysed very deeply as well. The analysis of the non-stationary effects are finally done taking into account the wind tunnel balance requirements and constraints, with special attention on an issue not commonly mentioned, the inertia forces generated on the balance by the model vibrations, and their influence on the aerodynamic forces to be measured. Several mentions to signal processing and acquisition are done, as this is the other key point on the measurements accuracy. However, it is easy to extrapolate these procedures to other types of balances, as the main intention is to show which are the critical points that make wind tunnel balances such a special and complex hardware.

We do not intend here to describe the design and calibration procedures of the industrial manufacturers. This is the result of a work done in the Universidad Politécnica de Madrid (UPM), and the Instituto Tecnológico y de Energías Renovables (ITER, Tenerife, Canary Island, Spain, www.iter.es). Nevertheless, we do consider that is a good guide for developers of wind tunnel balances in institutions like UPM and ITER, where research and education are very important points. 


\section{Wind tunnel balance}

The wind tunnels main function is to provide flow simulation on a model introduced in a fluid flow. Global forces and momentums on the model are mainly obtained by using different wind tunnel balances; although in special tests, local balances or pressure distribution measurement can be used as well. Range, accuracy and response time of the measurements are the main parameters that define such systems. The wind tunnel balances are extensively used and are an accurate method for measurements acquisition, with a wide range of measures and a fast response to loads changes. This system requires an important initial calibration effort but once the measurements are probed to be correct, the system can be used to test several low cost models with a reduced effort. Other option for aerodynamic load measurements is the pressure measurement in several model points by means of a pressure scanner or scanivalve system. This system requires a very complex and expensive test model. The measurement points are built in the model surface by making holes and connecting them with the scanivalve by means of a tube that transfer the pressure. These holes introduce also modifications in the flow around the model thus modifying the real behaviour of the model.

There are several types of wind tunnel balances. The most important are:

- External balances: They are placed outside the model, inside or outside the wind tunnel chamber test section, but they always introduce some interference in the wind flow. However the possibility to change test models with almost no effort provides a high flexibility to the wind tunnel facility. There are several degrees of complexity for these balances, depending mainly on the number of measurement channels, which can vary between 1 and 6 .

- Internal balances: They are placed inside the model, thus no interferences are introduced in the wind flow by the balance components, but a mechanical support for the model is always needed to maintain it in the test chamber and change the model orientation if desired. The complexity of the test model is comparable or higher than the models for scanivalve systems, as the balance has to be installed inside. Thus this option does not provide flexibility in testing different models. These balances are normally supplied to the customer already calibrated and with the acquisition system. The number of measured components can also vary between 1 and 6. Figure 1 shows an example of internal balance.

- $\quad$ Rotary balances: Used for propellers, helicopter blades and other rotating models.

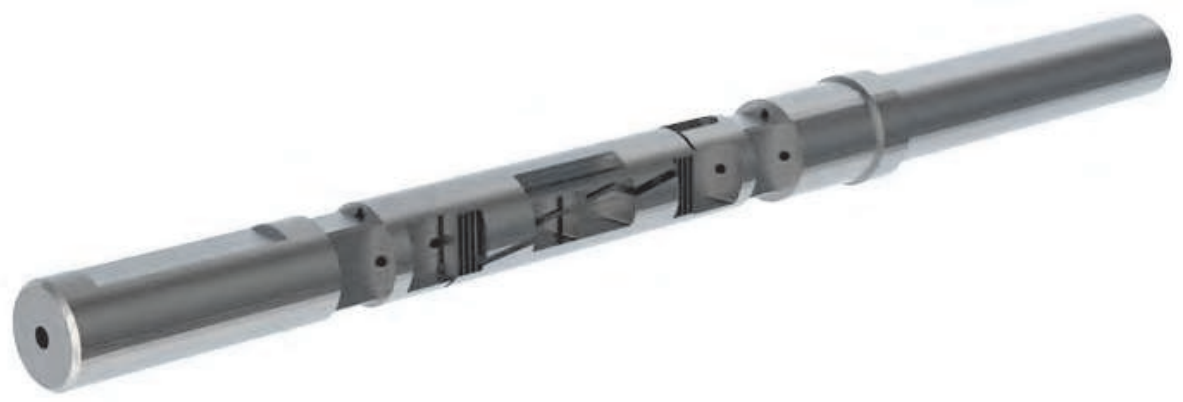

Fig. 1. Internal balance example. Image courtesy of STARCS. 
We now focus our attention in the 6 components external balances, as they provide 3 forces and 3 momentums measurement and a high flexibility for a multi test wind tunnel. Nevertheless the results presented in this report can be applied to almost all types of balances.

\subsection{Six components external balance}

There are several wind tunnel balances manufacturers, who have solved the problems presented in section 3 and they produce internal, external and rotary balances from the range of some Newtons up to some thousands of Newtons. But expensive commercial balances are not the only option to get an accurate wind tunnel loads measurement system. Taking into account our university environment, we, together with the ITER (www.iter.es), decided to manufacture our own balance for the ITER wind tunnel. The following chapters will show, as example to clearly explain the already mentioned key points, our experience, the problems we found during the calibration process and the solutions we have implemented, that will be beneficial for those who intent to build its own balance, as well as for those who want to understand the complexity of wind tunnel balance systems.

The commercial balances are used to have 6 complete coupled channels and a $6 \times 6$ decoupling matrix is provided by the vendor. This means that the forces and momentums measurements are obtained through a complex combination of the 6 channel signals by the use of a completely full decoupling matrix. The main inconvenient of this concept is that a recalibration of the balance requires a special calibration facility that is very expensive (see Figure 2).

The opposite balance design concept is a complete mechanical decoupling of the 6 loads components. Each channel output will be a unidirectional load signal (tractioncompression), and the composition of the signals will be the lift, drag, lateral forces and the pitching, balance, yawing momentums. One possibility could be to use three vertical sensors to obtain the lift force (which is the dominant force in aeronautical applications), the pitching momentum and the balance momentum, two horizontal sensors in the wind flow direction to acquire the drag force and the yawing momentum and one horizontal sensor perpendicular to the flow direction to measure the lateral forces. The calibration of these balances is much easier as the matrix is almost diagonal. Nevertheless, in practice the sensors absorb lateral forces and this results in a certain coupling effect. This coupling is particularly relevant between lift and drag.
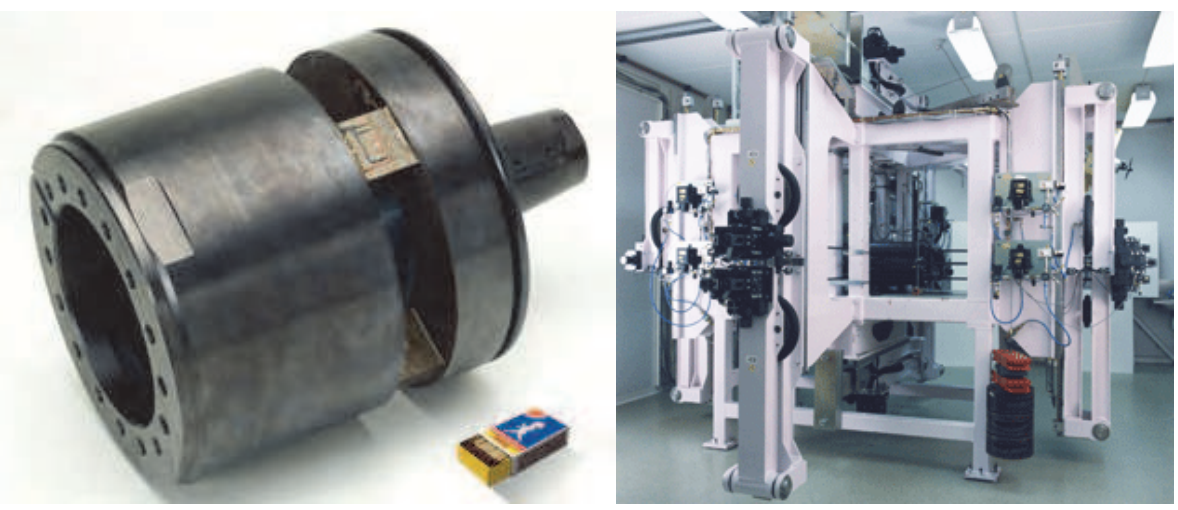

Fig. 2. External balance example on the left. Calibration facility example on the right. Images courtesy of STARCS. 
Besides the model supports, a balance support is needed to fix the balance to the wind tunnel structure. These supports are placed outside the wind tunnel test chamber, so their dimensions and weight are not important, the requirement imposed on them is an elevated stiffness.

Also important is to notice that once the external balance is providing good results for aerodynamic purposes, which are very exigent, it will provide also good results for most of other test models, as per example, cars, cyclists, buildings... so the use of this type of balance provides a multi-purpose test facility.

\subsection{Data acquisition components}

The data acquisition system (DAQ) is compounded by several items that allow the conversion of physical forces into digital values that can be managed by a computer. Several components comprise the DAQ and the selection of their properties will have an important impact on the system behaviour. The main components are the following:

a. Mechanics: The mechanics have to ensure that no friction forces are appearing thus and such not desired loads are avoided.

b. Sensors or transducers: The sensors are devices that convert the physical load in an electrical signal. The capability of the sensors to measure the force depends on the properties of the load and the sensor itself. Several types of transducers exist (Gorlin \& Slezinger, 1964):

- Weight balanced sensors

- Pneumatic and hydraulic sensors

- Electromagnetic sensors

- $\quad$ Spring sensors

- Strain-gauge sensors: These are very common transducers, also called load cells, which generate the electrical signal trough the mechanical deformation that causes the applied force. Usually 4 strain gauges in a Wheatstone bridge convert the deformation into electrical signals. There are many construction types but the $\mathrm{Z}$ shape ones have demonstrated in our own manufactured balance, that they are sensitive and accurate, with a relatively high stiffness.

c. Electronic amplifiers: The sensor output signal is a weak signal of around a few millivolts and its power has to be increased by means of an amplifier in order to have a distinguishable signal in the analog to digital converter.

d. Wires: The electrical signal is transferred from the sensor to the amplifier and from the amplifier to the sensor by means of wires, which are desired to be of high quality and efficient.

e. Analog to digital converter (ADC): These devices convert a continuous analog signal (voltage or current) into a discrete time digital signal. Several parameters differentiate the ADC, the most important ones are the resolution, the sampling rate and the accuracy. As demonstrated in step 3.1, an ADC with a resolution of 16 bits (216 analogto-digital conversion levels), 8 channels and a sampling speed of some $2.500 \mathrm{~Hz}$ per channel, result to fit with the wind tunnel balances requirements. Nowadays the hardware available at normal prices has enough quality to proportionate low error and thus good accuracy. Also the level of integration with computers is very high and manufacturers provide their own software.

f. Software: At the end of the data acquisition chain we encounter the software applications that post process the signal measurements in order to obtain the forces and 
momentums values. Several options are available, from commercial applications to homemade applications, from calculus sheets to complex programming codes, which have to be selected in function of the wind tunnel complexity.

\section{Wind tunnel balance calibration}

At this point, the importance of the wind tunnel balance calibration for eliminating errors in the measurement acquisition is obvious. The calibration, and recalibration if needed, is absolutely indispensable to eliminate the coupling effect and to determine the DAQ behaviour. Therefore the first step is to perform a static calibration, as explained below, and after that it is desirable to perform a dynamic calibration with the help of the already existent typical test models results.

\subsection{Static calibration}

The static calibration of the balance is not a trivial step in the chain to achieve reasonable system accuracy, although it could seem so in a first sight. As static suggest, this calibration can be done with a standalone balance, without the presence of the wind tunnel itself, and this is the standard procedure for industrial balance manufacturers. Making a calibration with the balance dismounted from the wind tunnel is much easier than calibrating it attached to its mounting supports on the tunnel. The accessibility to the balance to test every axe, in every direction and in both ways, in order to identify the behaviour of the acquisition system (mechanics, load cells, amplifier, wires, DAQ, software) is definitely easier.

Nevertheless, as shown in Figure 2, the calibration facility is very sophisticated and expensive. As this chapter treats about a self-designed and constructed balance, such calibration facility is not available, so another procedure is necessary. The gained experience shows that, in this case, instead of a standalone calibration, it is better and more accurate to do it with the balance installed in the wind tunnel. This calibration philosophy ensures that the reference axes of the balance are the same that those of the test chamber. In principle it can be though that once the balance is calibrated outside of the test chamber, it can be mounted properly by determining the local vertical and horizontal directions of the test chamber, but this may be a big mistake due to the following two reasons:

a. The balance mounting actions could ensure a good alignment between balance axes and test chamber axes, but in any case some deviation could be expected. Although these deviations are rather small, in the case of wind tunnel testing were require very high accuracy, they could be catastrophic for the test results. To show the importance of an angular deviation, let's assume that only a 1 degree deviation has occurred between balance and test chamber vertical direction. Let's assume also that the test chamber vertical direction corresponds with the lift direction and, correspondingly, the test chamber horizontal direction corresponds with the drag direction. The drag measurement is where there is a major impact on results, for example, if a $150 \mathrm{~N} \mathrm{lift}$ force is applied to the balance, around $2,6 \mathrm{~N}$ are incorrectly measured in the drag direction due to the misalignment. In a model of a medium speed aircraft, the drag corresponding to a $150 \mathrm{~N}$ lift would range from 10 to $12 \mathrm{~N}$, meaning that the measured drag value has a deviation of roughly $25 \%$, which is an unacceptable error for aerodynamic model tests. 
b. The wind flow direction inside the test chamber has to be determined. We cannot assume that the wind direction and test chamber horizontal direction are the same, as the risk of being committing a same order error as the presented in the previous step is not acceptable.

A correct wind tunnel balance calibration must be preceded by some steps that allow the determination of fixed and reliable axes. The first action has to be the installation of the balance in its fixed final position in the test chamber. The second action has to be an accurate determination of the local wind flow direction inside the test chamber.

The flow direction determination can be done with the help of different instruments; the most common are the cobra, the wedge, the five holes and the cylindrical probes. All of these probes based their detection method on the same concept. The probes are a bundle of tubes where the leading edge of one of them is perpendicular to the flow direction while the leading edge of the others, one or two pairs, are cut at a known angle to the flow direction. The probe is placed in the test chamber, making an angle with the flow direction, then the pressure measured in the external probes (see Figure 3) are different, and through a precalibrated curve or by reorienting the probe, the angle of the flow can be determined.

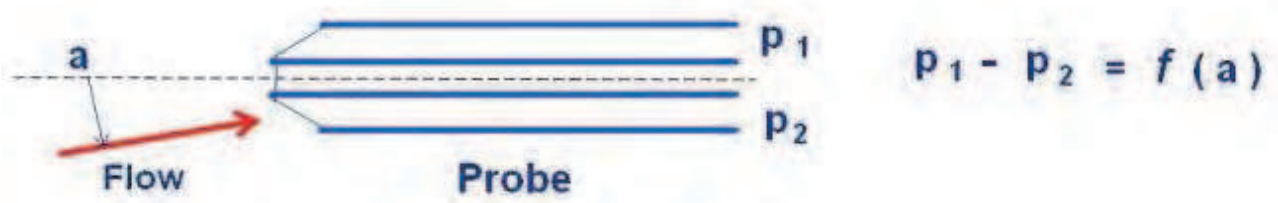

Fig. 3. Vertical cut of three tubes showing the leading edges and the angle determination concept

Both attack angle and yaw angle can be determined by using one probe (with two pair of external tubes) or two probes (with one pair of external tubes) (see Figure 4). The leading edge angle to the flow direction determines the sensitivity of the probes to the changes in the flow angle, the dynamic pressure or the Reynolds number (Glenn LTP NASA and University of Cambridge web pages).

Once the flow direction is fully determined, the lift and drag forces directions are now known. The lift force perpendicular and the drag force parallel to the wind flow. The axes in which the forces (and momentums) have to be measured are properly defined.

Once checked that the flow is horizontal, the system is now ready for the calibration process. As aforementioned, we do not have specific calibration facilities. To avoid this problem, we have implemented some special calibration procedures, which are time consuming, but they give very accurate results.

As the function of the balance will be the measurements of aerodynamic forces, the calibration method should introduce loads in the proper direction and sense. Thus, the loads are introduced in the lift direction and not in weight direction, as this is the most critical direction while working with low drag aerodynamic models (for other applications the load direction will be of no issue). In order to ensure that we are loading the balance exactly in the appropriate direction, a specific system has been design. 

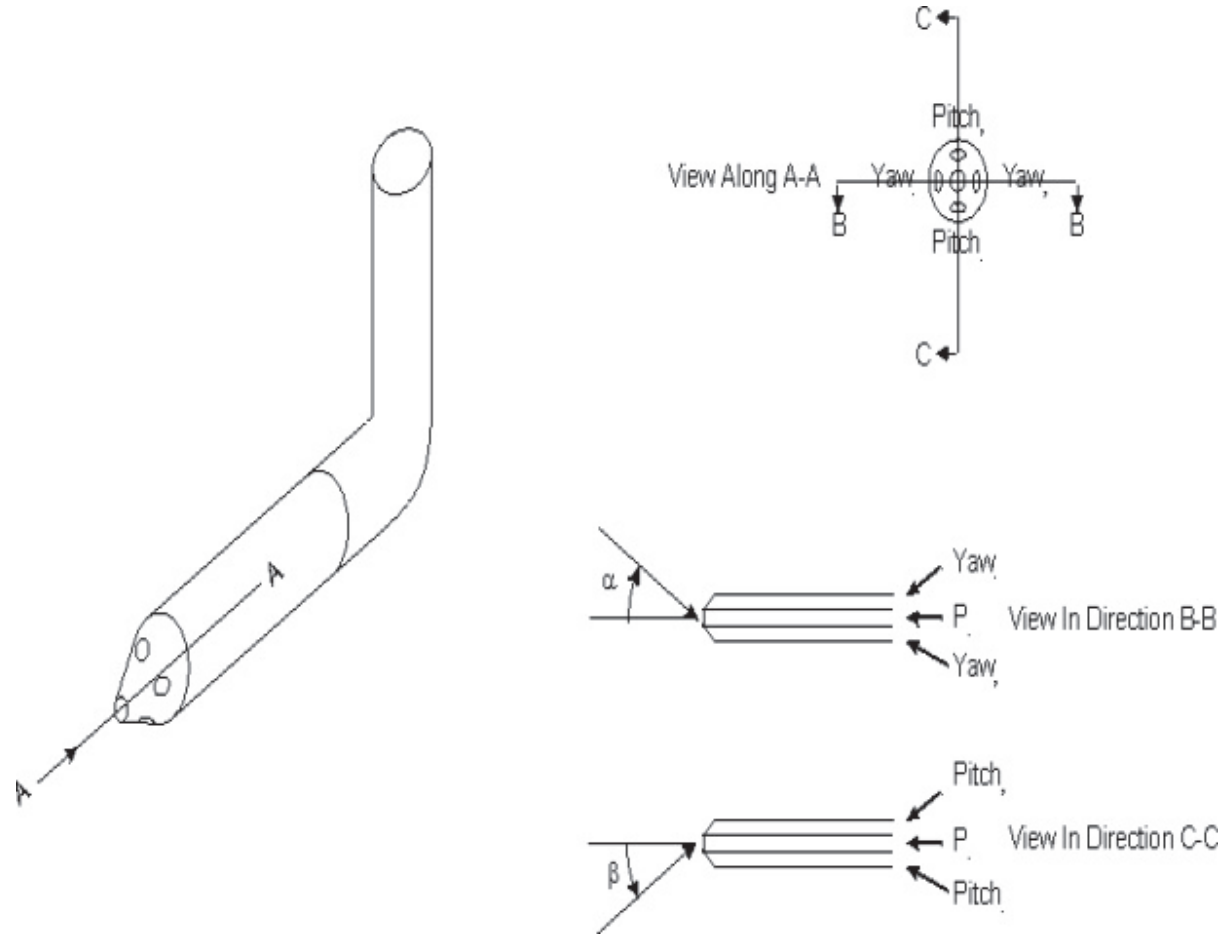

Fig. 4. A five hole truncated pyramid probe

The procedure to be shown here takes into account, that we use standard and calibrated load cells to measure the forces on each bar. Nevertheless, we apply known forces in the direction of the three axes to check not only the calibration of the load cells, but also the signal amplifier and the data acquisition system. As we expected, the manufacturer cell calibration and our calibration had a very good agreement, differences were less than $0,1 \%$. It probes as well, that our philosophy of mechanical decoupling is a very good approach for the design of external balances.

It is important to point out that, at this moment, we were interested only in one direction each time. So small errors in the vertical or both horizontal directions (wind direction and transverse) did not introduce important errors on the true loads, because the cosines of the expected deviation angles are practically 1,0 .

As abovementioned, for the current application, the only relevant coupling is between drag and lift. Taking into account that the flow in the test section is horizontal, an special device was designed to ensure that we apply a vertical load to the balance, with an error less than $0,1 \mathrm{~mm}$ in $600 \mathrm{~mm}$. In these conditions, the coupling can be measured and corrected, and for a lift coefficient of 0,5 , which is normal for cruise conditions of low speed aircrafts, the effect on drag is in the order of one drag count.

Figure 5 shows the results of the drag due to lift coupling tests, the $x$ axis represents the loads in the vertical direction (lift) and the $y$ axis the measured in horizontal direction (drag). It shows that the coupling effect is almost linear, being the drag $0,46 \%$ of the applied lift. 


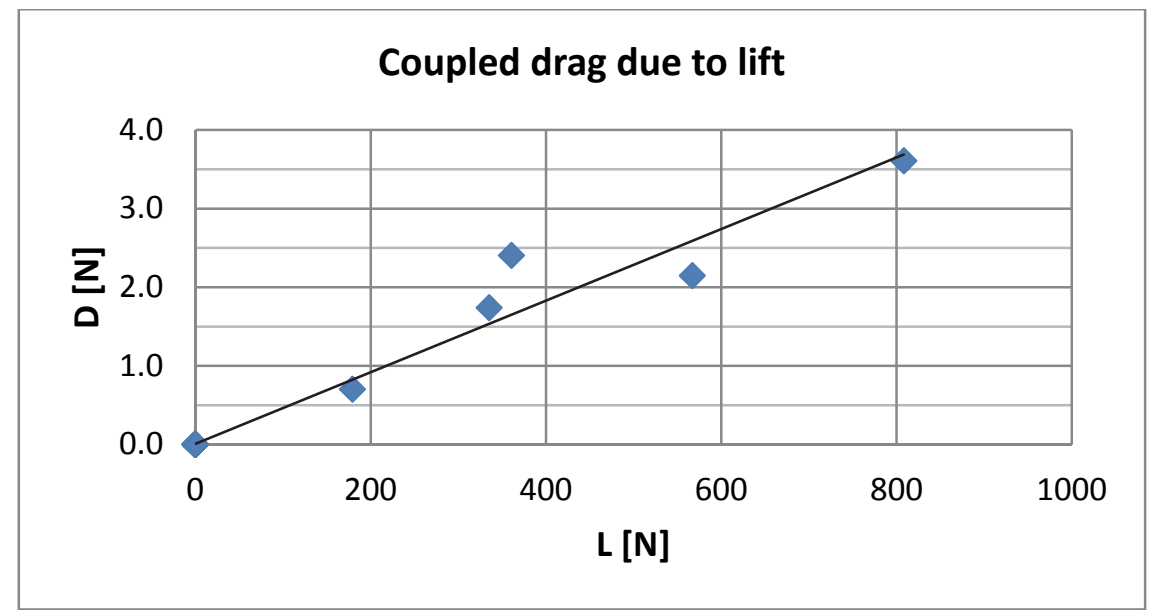

Fig. 5. Coupled drag due to lift. Horizontal axis represents the applied vertical force, and vertical axis the measured horizontal component due to coupling effect.

\subsection{Dynamic calibration}

Finally, to check all the system working in real conditions, we could use a reference model, such as a two dimensional model of the NACA 0012 wing section, to make final adjustment of the DAQ and coupling corrections. A well known standard calibration model would be another option, but the L/D ratio is well below the range we expect to make our tests. In both cases, differences in turbulence level and/or surface smoothness may produce differences of the same order than those to be checked.

Due to those reasons and the cost, in terms of time and money, it was finally decided to make a deep analysis of the measured results, to determine the DAQ requirements needed to get a high confidence level on the measured forces. The complete procedure is presented in the following paragraph.

\section{Wind tunnel balance requirements}

The better way to understand the strong requirements demanded to a wind tunnel balance is by studying the following example, where rough numbers involved in the problem are estimated.

In a 2,0x2,0 m low speed wind tunnel test section, with a testing velocity of around $50 \mathrm{~m} / \mathrm{s}$, using a model of a medium speed aircraft at cruise conditions, the lift would be roughly 150 $\mathrm{N}$ and the drag would range from $10 \mathrm{~N}$ to $12 \mathrm{~N}$, for the high aerodynamic efficiency condition. For this type of model aircraft, the accuracy of the drag coefficient must be greater than 0,5 drag counts, which means that the accuracy of the balance, in the drag direction, must be higher than $0,015 \mathrm{~N}$.

For the case of static loads measurements, where there is only one component, accuracy may or may not be a very tight requirement. But in the case of the wind tunnels, where we intend to measure simultaneously up to 6 components, forces and momentums, on a model under aerodynamic loads, with some non-stationary terms due to vibrations; amongst others, the following problems can be expected: 
a. The model vibrations induces inertia forces on the balance.

b. There are aerodynamic forces on the model support system and interferences between these and the model may appear.

c. The complete mechanical decoupling of the forces components is very difficult and expensive; therefore some coupling between lift and drag may appear.

During the setting of a new self-designed balance, all these points have been deeply treated, and the acquired experience is the base for this paragraph.

\subsection{Inertia loads on the balance}

When testing an aerodynamic model in a wind tunnel, the forces and momentums measured come from different sources. The most important ones are the aerodynamic forces and momentums themselves, but some other undesirable loads could be expected. Although wind tunnel balances are necessarily very stiff, due to the model support and other elements, it is difficult to avoid oscillatory movements of the model, due to the aerodynamic loads. Those vibrations induce inertia forces and momentums on the balance, and might have a negative impact in the validity and/or accuracy of the measurements. Also some intrinsic data acquisition system noise has to be expected. Inertia forces and electronic noise are not desired, but measured, together with the aerodynamic loads; as those are not present in a free flight model, the study and evaluation of their impact on the pursued results is very important.

The inertia loads that appear in wind tunnel tests will depend strongly on the test facility and its components, so a specific study of each configuration should be performed. Nonetheless, the procedure, the critic analyses of the measurements and the influence range of these not desired loads are similar for all the wind tunnel tests configurations, so the results presented in these paragraphs will be of high interest for wind tunnel test engineers. We can start making some rough calculations to show the true magnitude of the inertia loads. If we consider an amplitude in the order of 0,5 millimetres and an oscillatory frequency in the flow (drag) direction of some $5 \mathrm{~Hz}$, which are very representative true values, the oscillation of the model produces accelerations in the range of $0,49 \mathrm{~m} / \mathrm{s}^{2}$. If the mass of the model is, i.e., $50 \mathrm{~kg}$, the appearing inertia forces are of the order of $24 \mathrm{~N}$, which is of the same order of magnitude as the value of the drag that is to be measured. This is the reason why these inertia loads have to be carefully studied, in order to determine their magnitude, shape and relation with the aerodynamic loads.

First point to be determined is the sampling rate of the electronic signal, which is necessary for an accurate determination of the inertia terms, and thus the determination of the true aerodynamic signal. The aliasing, which is an effect that cause different sampled signals to become indistinguishable, is taken as the reference for the sample rate selection in several publications. The sampling theorem, frequently called Nyquist sampling theorem, states that a continuous signal can be properly sampled if the following expression accomplished (Smith, 1997):

\section{Sampling Frequency $>2$ * Band Wide}

Following this rule, if we have a frequency signal of around $5 \mathrm{~Hz}$, it can be stated that a sample rate of more than $10 \mathrm{~Hz}$ is enough for our purposes. As we will show, this is not enough in our case, due to several reasons, between them: the signal frequency study will not be accurate and the sample rate will impact the measurement results. The sampling rate 
should be high enough to get accurate results and avoid the previous issues. Nowadays, the sampling frequency of the wind tunnel balance systems is much higher than the maximum expected frequency of the signal induced on the balance by the aerodynamic and inertia forces, thus those mentioned problems are overpassed, as well as the aliasing effect. Also with today's computer capabilities, managing an elevated quantity of data is no longer a problem. High sampling frequency produces benefits in reducing the random noise originated in the acquisition system (DAQ) as well.

While using low sampling rates, it is important to ensure that the sampled signal comprises complete cycles, so that no partial cycles are included in the signal mean and standard deviation calculation. The values obtained if, as an example, a half wave is included, could cause significant deviations with relation to the correct results. However, this effect is only recognizable using low sapling rates, and disappears if a high sampling rate is used.

In order to ensure that the noise of the measured signal is low enough for our purpose, the signal to noise ratio (SNR) has to be higher than 3, condition that ensures that every signal is recognizable. Although this is a soft requirement to the hardware currently available, there is always present a noise signal that is a not desired random signal. One simple and efficient method that can be applied to cancel out the noise signal is the time-averaging. This method, as presented in several publications (Lohninger, H., 2010), consist on registering and summing up the signal repeatedly, an action that applied to a stable signal eliminates the noise signal (our aerodynamic loads and inertia loads are supposed to be stable over hundreds of cycles once the system reaches a steady state). A high sample rate impacts positively in this noise reduction method.

Before starting aerodynamic tests, some mechanical tests were done to determine the main frequency of the complete system, balance plus model. Two free vibration movements were induced, in the flow direction and in yaw oscillation. In both conditions, the reactions on the balance were measured. The signal analyses showed that the main frequency for such unforced movement was, roughly, 5,2 Hz. In principle, this is the main frequency to be considered for the following analysis, and it will be shown that this main frequency is not modified by forced oscillations, due to aerodynamic loads.

The determination of the minimum sampling rate that provides accurate results has to take into account several considerations. The most important ones are, as already mentioned, the accuracy of the results and the main inertia frequency determination. The first measurements acquisitions, although the main inertia forces frequency is already known, have to be taken with a high sampling rate, at a minimum of 2 orders of magnitude above the expected vibration frequency (even 3 orders of magnitude are recommended for the complete analysis). As explained later on in this text, this will allow a proper frequency domain study, as no frequency components are lost due to a low sampling rate. The focus of attention is the accuracy of the results. The sampling rate has an important impact in the mean of the measured signal, which, at the end of the process, is the aerodynamic load we want to measure. Through the following example the influence of the sampling rate is clearly shown. Figure 6 shows the mean value of a real wind tunnel signal calculated each second for total signal duration of 20 seconds (blue line). The signal is the same for all the plots and the only difference is the sampling rate, from $2.500 \mathrm{~Hz}$, near 3 orders of magnitude above the mechanical main frequency, to $10 \mathrm{~Hz}$, double of the main frequency. The linear regression of those average values is also plotted in all the figures. The last plot is the representation of the whole average signal mean value, for the complete 20 seconds, versus the sampling rate. 
As shown in Figure 6, for low sampling rates, $10 \mathrm{~Hz}$ and $20 \mathrm{~Hz}$, which have the same order of magnitude than the Nyquist sampling rate for this signal, the accuracy and reliability of the mean value is poor. The linear regression and the mean value have important deviations. However, a sampling rate of $100 \mathrm{~Hz}$ gives good results although this frequency, as shown in the plot of the averaged force mean value, is at the limit. For higher sampling rates as $500 \mathrm{~Hz}$ and $2500 \mathrm{~Hz}$ the accuracy of the force mean is very good.
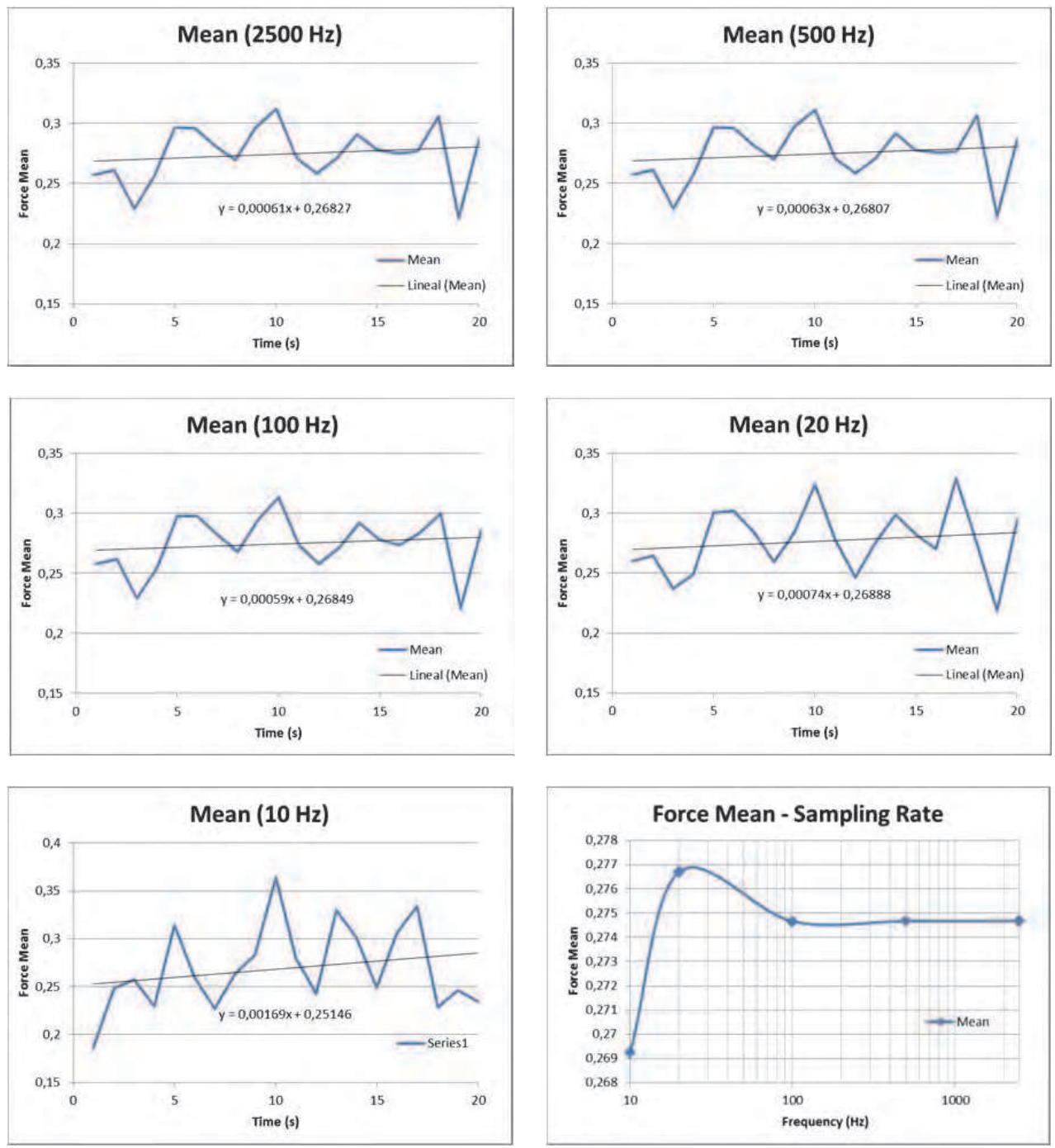

Fig. 6. Plots of a signal force mean calculated each second. Five plots for different signal sampling rates: $2.500,500,100,20$ and $10 \mathrm{~Hz}$. Plot of the whole averaged force mean value versus sampling frequency. 
Then, it can be stated that the minimum sampling rate to get accurate results has to be at least 2 orders of magnitude above the Nyquist sampling rate. Even if the sampling rate used were very high, it is always recommended to perform this study, to understand the complete phenomena that occurs.

Once the appropriate measurements are acquired, the signal properties can be studied. It is interesting to know the frequency components of a signal (in example the inertia forces frequency) but their identification by its representation in the time domain usually is a difficult task. A deeper study of the measured signals trough the Fourier transform provides the frequency components of the signal. One fast approach to this Fourier transform study is to perform a discrete Fourier transform, which is identical to samples of the Fourier transform at equally spaced frequencies, by using the fast Fourier transform method (FFT) (Oppenheim \& Schafer, 1989). In the frequency domain, it is easy to identify each of the different signal sources. With the aim to clearly show the influence of each measurements source, real measurements will be presented below. Those were taken at the ITER wind tunnel, that is provided with an own manufactured balance, which is an accurate wind tunnel 6 mechanically decoupled components balance.

ITER Low Speed Wind Tunnel (ITER-LSWT) has a 2,0x2,0 $\mathrm{m}$ test section, 3,0 m long. In the current configuration, which includes devices to reduce turbulence, the maximum allowable speed is $50 \mathrm{~m} / \mathrm{s}$, with a turbulence level below $0,5 \%$. The model used for the tests is a section of a wing, still under design, to be used in a solar powered aircraft, with $667 \mathrm{~mm}$ cord and $1.990 \mathrm{~mm}$ span.

It is important to look at the signals frequency spectrum from a critical point of view. It is not enough to identify the main frequency but the amplitude spectrum of the frequencies, and their importance in comparison with the constant signal $(\sim 0 \mathrm{~Hz}$ frequency signal) that represents the aerodynamic force acting on the model. This is clearly shown in Figure 7 , were the signal is plotted in both: time and frequency spectrum.

The main frequency of the inertia forces signal is slightly higher than $5,0 \mathrm{~Hz}$, the same than for the mechanical free vibration, and its amplitude value in comparison with the complete signal amplitude is near $75 \%$.

Most of the balances obtain the forces and momentums values from a combination of several load cells measures. Then, it can be thought that the study of the resultant load could be enough to determine the inertial behaviour of the system, but this is not true. It is important to study the frequency behaviour of each measurement channel signal, before studying the combination of the measurement channels. Figure 8 shows the resultant signal of the two cells that measure the drag component in the ITER-LSWT balance. In this case, although there is a pick of frequency for the same value than the single analysis, it should be stated that the main inertia movement is occurring at around $10 \mathrm{~Hz}$.

It is clearly shown that in the combined signal, although a small peak appears at 5,0 $\mathrm{Hz}$, the main vibration frequency is $10 \mathrm{~Hz}$. However, Figures 9 and 10 show the 2 measured signals whose combination result in the previous drag force signal.

This happens because of several coincidences: the model is symmetric, both load cells are placed equidistant along the symmetric model and the inertial vibration axe induced on the model by the aerodynamic flux is perpendicular to the load cells plane. Thus, when both channel signals are summed up, the inertia components are cancelled. This is a very good example to emphasize the necessity to study signal channels independently, prior to forces and momentums calculation, although the extreme case, with other model or in a different wind tunnel facilities configuration this behaviour could not happened. 

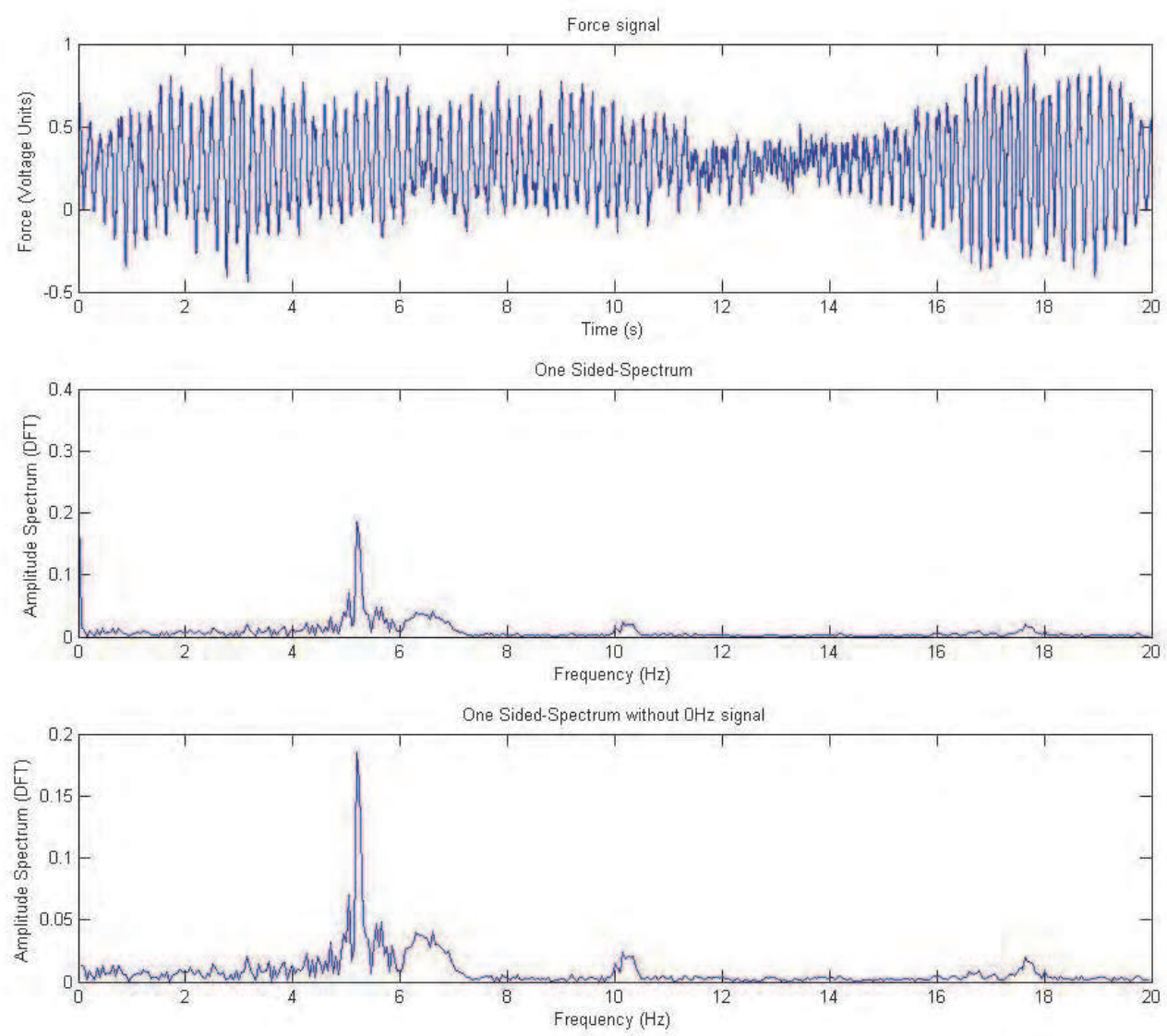

Fig. 7. Representation of a measured signal, in voltage, at $2.500 \mathrm{~Hz}$ sampling rate of an aerodynamic model in the ITER-LSWT, at a wind speed of $20 \mathrm{~m} / \mathrm{s}$ and at angle of attach of $8^{\mathrm{o}}$. Time domain signal plot during $20 \mathrm{sec}$. Frequency domain signal plots with different amplitude scales, with and without the $0 \mathrm{~Hz}$ component of the signal. 

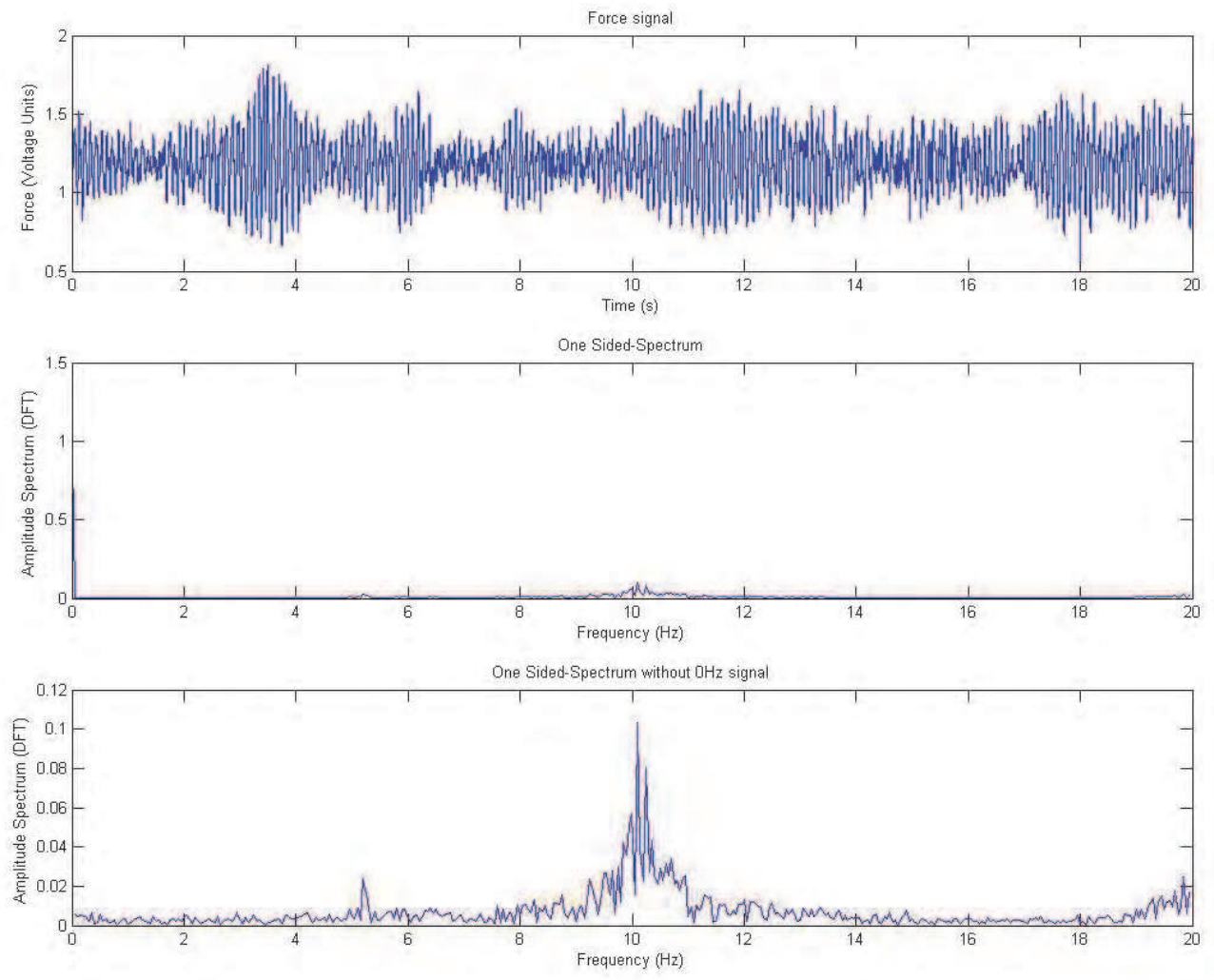

Fig. 8. Representation of a resultant force signal, combination of two drag cells, at a $2.500 \mathrm{~Hz}$ sampling rate of an aerodynamic model introduce in the ITER-LSWT, at a wind speed of 40 $\mathrm{m} / \mathrm{s}$ and at angle of attach of $8^{\circ}$. Time domain signal plot during $20 \mathrm{sec}$. Frequency domain signal plots with and without the $0 \mathrm{~Hz}$ component of the signal. 

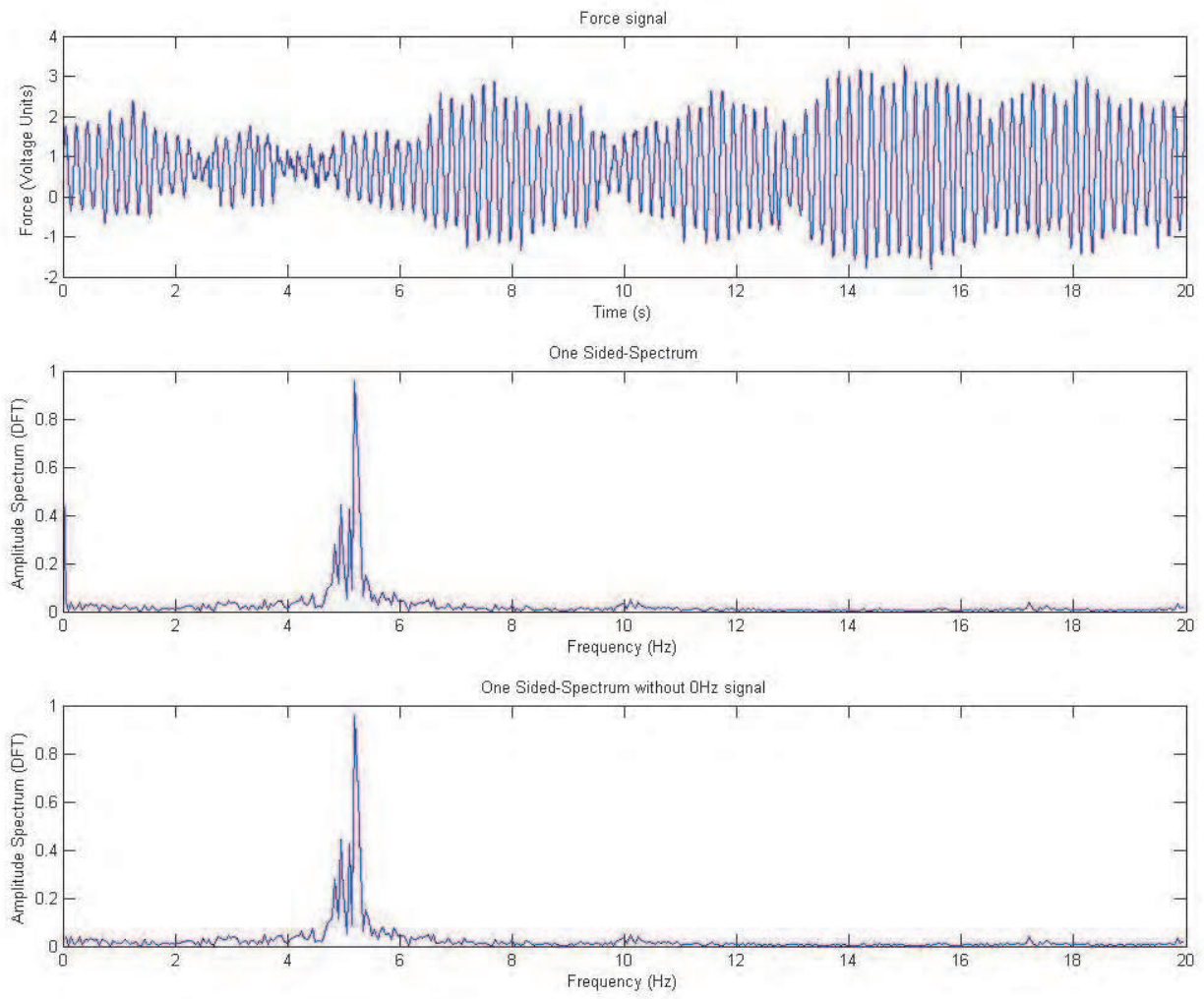

Fig. 9. Representation of channel 1 signal at a $2.500 \mathrm{~Hz}$ sampling rate of an aerodynamic model introduce in the ITER-LSWT, at a wind speed of $40 \mathrm{~m} / \mathrm{s}$ and at angle of attach of $8^{\circ}$. Time domain signal plot during $20 \mathrm{sec}$. Frequency domain signal plots with and without the $0 \mathrm{~Hz}$ component of the signal. 

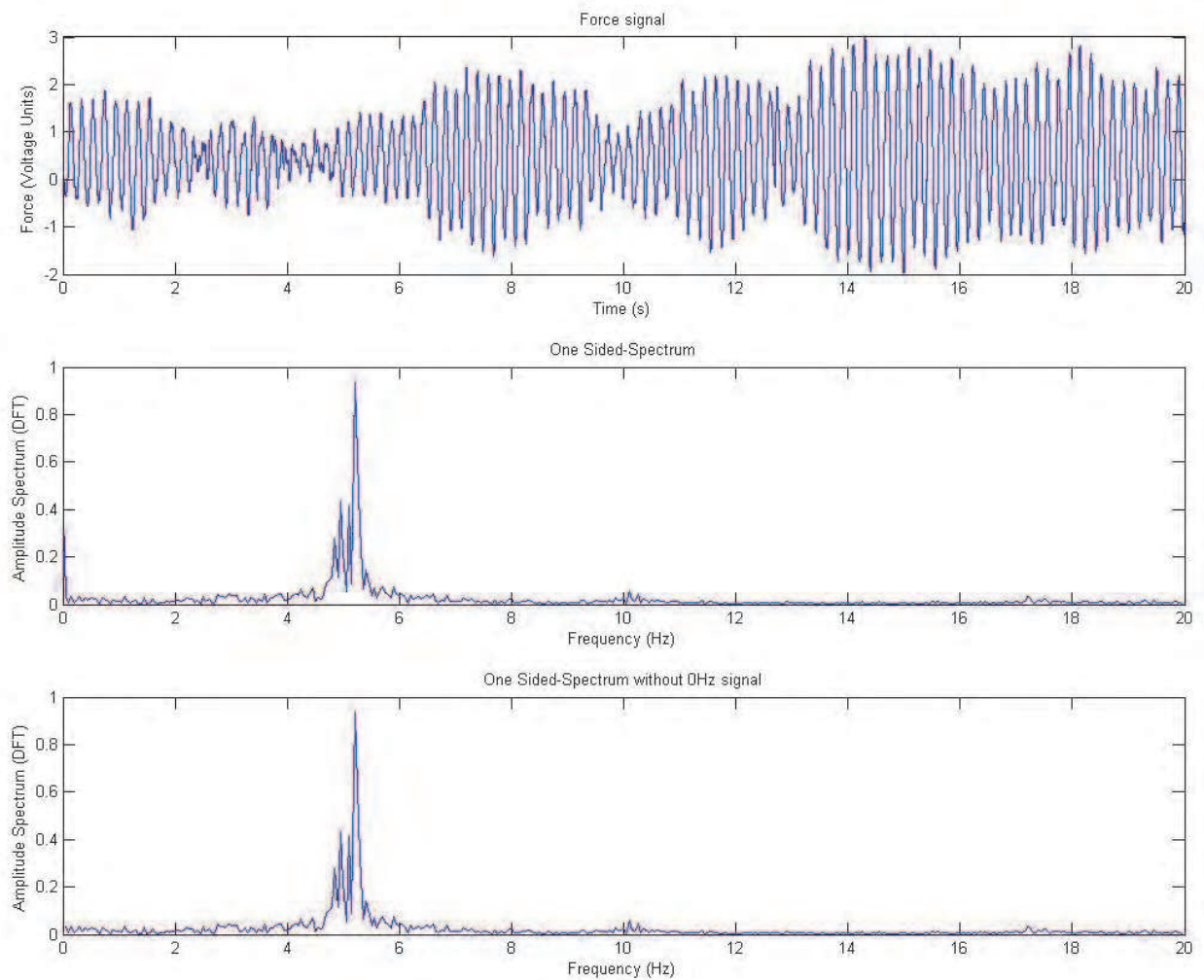

Fig. 10. Representation of channel 2 signal at a $2.500 \mathrm{~Hz}$ sampling rate of an aerodynamic model introduce in the ITER-LSWT, at a wind speed of $40 \mathrm{~m} / \mathrm{s}$ and at angle of attach of $8^{\circ}$. Time domain signal plot during $20 \mathrm{sec}$. Frequency domain signal plots with and without the $0 \mathrm{~Hz}$ component of the signal. 
Due to the particular characteristics of aerodynamics models, the influence in the drag measurements is much more important than in the other forces and momentums, such as the lift measurements, so it is highly recommended to focus the attention in those channels related to the drag force measures.

\subsection{Model support interferences}

The external balances are used as fix element of the wind tunnels, however the test models are interchangeable items. The model support system is the mechanical link between the balance and model. There are several types and sizes of supports, but all of them may interfere with the aerodynamic measurements. Two origins of interferences can be highlighted:

a. Aerodynamic forces on the model support system.

b. Aerodynamic interference between the model support system and the model itself.

The occurrence and impact of each of the interferences depend on the shape, size and disposition with relation to the model, but in almost all cases, mainly in aeronautical purposes, the model support system is another source of problems for an accurate drag measurement.

A direct model fixation to the balance might be the best option in order to avoid interferences, but this solution will work in few and very specific cases, such as, half wings or half model aircraft. In all other cases of interest, as wings, nacelles, complete aircrafts... tests, the presence of a more or less complex support cannot be avoided; as the model has to be placed more or less in middle of the test chamber, and the aerodynamic forces have to be transmitted to the balance. Moreover, it is very common that for aeronautical tests, as a minimum, the angle of attack of the model should be variable, which introduce more complexity to the support and, therefore, more interferences.

Excluding a few cases, a support system is unavoidable. Its influence quantification and the methods to subtract the interferences are key points to gain measurement accuracy. If a very stiff support is used, its drag and interferences on the model may be very important, once more in the order of the values to be measured for cruise conditions. On the other hand, the size of the support fairings will be smaller if we just faire the supports, but in this case the supports drag becomes very important too.

There are several methods to subtract the contribution of the support to the balance forces and momentums measurements. Three methods with a complete different concept are presented here:

- Experimental method: By taking series of measurements before placing the model, it is possible to determine the support aerodynamic contribution. However, by means of this method, the possible aerodynamic interferences between support and model are not contemplated. In case the supports wake does not impact on the model, and vice versa, the mutual influence would be negligible, but in other case, the supportmodel interference might be studied with more detail. This is a very common method, as other none desired loads are eliminated as well, from the model and support weight.

- Theoretical method: Theoretical calculations of aerodynamic forces and momentums over the supports can be subtracted to the wind tunnel measurements. The supports 
fairing geometry should be known and common, with an extensive and contrasted bibliography, so that the aerodynamic forces and momentums theoretic calculus will be reliable enough. The support fairings airfoil should be a symmetric one, and its angle of attack should be $0^{\circ}$ in order to generate only drag force and one momentum component. Also, the possible interferences between support and model and vice versa have to be assessed.

- Hybrid method: It is a mix of both previous methods, providing an easier experimental part and a more reliable theoretical part (Horsten \& Veldhuis, 2009).

Once support forces and momentums has been measured or computed, it is convenient to obtain the aerodynamic forces and momentums coefficients, made non-dimensional using the same characteristic surface and dimensions than for the model, in order to be able to substrate those coefficients to the measured ones. It is also convenient to test the supports for the same Reynolds number $(\mathrm{Re})$ range than the model to be tested, then it should be possible to obtain a fitted curve for each coefficient, normally depending upon $\log (\operatorname{Re})$.

\subsection{Coupling effects}

The balance that has been used in the ITER-LSWT facilities was designed with the philosophy of mechanical decoupling of forces. So that, the measurement of loads on six traction-compression bars allows obtaining all forces and momentums components. From the theoretical point of view, this design method supposes that there is no coupling in the three forces components, because bars cannot support any transverse load. Nevertheless, it is not absolutely true, and some coupling effects among measured loads must be expected. There are several sources that could contribute to the coupling effect. The most relevant are:

a. Deformations of the balance forces transmission rods.

b. Deformation of the model supports.

c. Inaccurate assembly of balance components.

These errors can be eliminated by means of a correct calibration, as presented in step 4, but it is important to know their magnitude in order to stress the importance of this calibration.

This coupling effect is very critical in the relation between lift and drag. In a high quality balance such coupling is only about $1 \%$, which could seem to be negligible, but, in the case of aeronautical applications, this is the order of the ratio between lift and drag, so that such coupling must be accurately measured during the calibration process. If we consider the example introduced at the beginning of this section, a lift force of around $150 \mathrm{~N}$, may produce a force of $1,5 \mathrm{~N}$ in the drag direction, that is in the same order of magnitude than the drag forces appearing due to aerodynamic loads.

\section{Conclusions}

The first tests done with our self-designed and manufactured balance has been on a two dimensional profile. Such profile will be used in a solar powered aircraft, with critical design conditions for $\operatorname{Re}=500.000$ and lift coefficient, $c_{l}=0,8$ and with constant cambered upper surface, in order to facilitate the installation of the solar cells. Profile aerodynamic characteristics were computed using several codes previous to its manufacturing and 
testing, because a minumum aerodynamic efficiency of 80 is required in the abovementioned design conditions. Experimental results have shown a complete agreement with the expected ones, including lift curve slope and aerodynamic efficiency, proving that both, balance capabilities and calibration procedures, are good enough for our requirements: a maximum error of one drag count for critical design conditions. Of course, many balances achieve errors even one order of magnitude less, but their cost is several orders of magnitude higher.

From the technical point of view, we have learned many things, but we want to emphasize only three important aspects:

- Mechanical decoupling is a very efficient design philosophy to reduce sources of errors and to facilitate balance calibration.

- Self-designed and manufactured wind tunnel external balances should be calibrated mounted in the definitive place.

- Detailed measurement and analysis of the measured signal is highly recommended to fix DAQ performances, and can efficiently replace the dynamic calibration.

\section{Acknowledgment}

The whole activities have taken about ten years, with the participation of many professionals and students. First of all, authors wish to acknowledge to ITER and its managing director, Manuel Cendagorta, the support given in balance manufacturing, setting and tests. In particular, we must mention to Esther Friend, because of her personal compromise and enthusiast help; the support given by Guillermo Galván, Jesús Fernández, Jesús Rodríguez, Juan Enrique Martín, and many others.

Many thanks as well to Lidia Esteban and Alvaro Lozano, students of the UPM, which have collaborate in different times of the project. The latest, but not the less important, students involved in the project have been Artur Jarzabek and Christopher Field, from the Imperial College; thanks in deed for your help in final tests performance and proof reading.

\section{References}

Gorlin, S.M. \& Slezinger, I.I. (1966). Wind Tunnels and Their Instrumentation, Israel Program for Scientific Translations, No 1680, Israel, Jerusalem

Horsten, B.J.C. \& Veldhuis, L.L.M. (2009). A New Hybrid Method to Correct for Wind Tunnel Wall- and Support Interference On-line, World Academy of Science, Engineering and Technology 58

Lohninger, H. (2010). Fundamentals of Statistics, Ihr Epina eBook-Team, Retrieved from <http://www.statistics4u.com/fundstat_eng/>

Oppenheim, Alan V. \& Schafer, Ronald W. (1989). Discrete-Time signal Processing, PrenticeHall Inc., ISBN 0-13-216776-9, USA, New Jersey

Smith, Steven W. (1997). The Scientist and Engineer's Guide to Digital Signal Processing, California Technical Publishing, Retrieved from

< http://www.dspguide.com/>

Unknown author (2010). Pressure Probes, Department of Engineering, University of Cambridge, Retrieved from

http://www-diva.eng.cam.ac.uk/whittle/pressure. html 
Various authors (2010). Beginner's Guide to Wind Tunnels, Glenn Learning Technologies Project ; NASA, Retrieved from

<http://www.grc.nasa.gov/WWW/k-12/ airplane/bgt.html > 


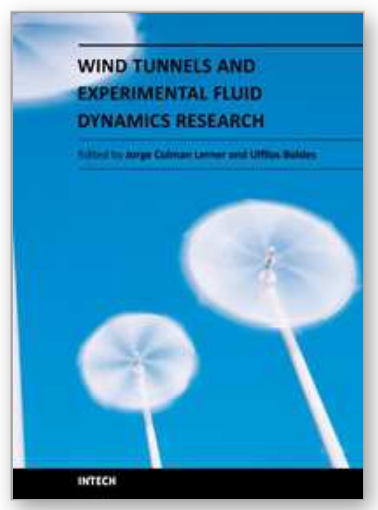

\author{
Wind Tunnels and Experimental Fluid Dynamics Research \\ Edited by Prof. Jorge Colman Lerner
}

ISBN 978-953-307-623-2

Hard cover, 709 pages

Publisher InTech

Published online 27, July, 2011

Published in print edition July, 2011

The book â€œWind Tunnels and Experimental Fluid Dynamics Researchâ€ is comprised of 33 chapters divided in five sections. The first 12 chapters discuss wind tunnel facilities and experiments in incompressible flow, while the next seven chapters deal with building dynamics, flow control and fluid mechanics. Third section of the book is dedicated to chapters discussing aerodynamic field measurements and real full scale analysis (chapters 20-22). Chapters in the last two sections deal with turbulent structure analysis (chapters 23-25) and wind tunnels in compressible flow (chapters 26-33). Contributions from a large number of international experts make this publication a highly valuable resource in wind tunnels and fluid dynamics field of research.

\title{
How to reference
}

In order to correctly reference this scholarly work, feel free to copy and paste the following:

Miguel Gonzalez, Jose Miguel Ezquerro, Victoria Lapuerta, Ana Laveron and Jacobo Rodriguez (2011). Components of a Wind Tunnel Balance: Design and Calibration, Wind Tunnels and Experimental Fluid Dynamics Research, Prof. Jorge Colman Lerner (Ed.), ISBN: 978-953-307-623-2, InTech, Available from: http://www.intechopen.com/books/wind-tunnels-and-experimental-fluid-dynamics-research/components-of-awind-tunnel-balance-design-and-calibration

\section{INTECH}

open science | open minds

\section{InTech Europe}

University Campus STeP Ri

Slavka Krautzeka 83/A

51000 Rijeka, Croatia

Phone: +385 (51) 770447

Fax: +385 (51) 686166

www.intechopen.com

\section{InTech China}

Unit 405, Office Block, Hotel Equatorial Shanghai

No.65, Yan An Road (West), Shanghai, 200040, China

中国上海市延安西路65号上海国际贵都大饭店办公楼 405 单元

Phone: +86-21-62489820

Fax: $+86-21-62489821$ 